\title{
Calidad del agua de la niebla Captada artificialmente en la microcuenca del río Pixquiac, Veracruz, México: RESULTADOS PRELIMINARES
}

\author{
Rodolfo Jofre-Meléndez ${ }^{1}$, Juan Cervantes-Pérez ${ }^{1}$ y Víctor L. Barradas ${ }^{2}$ \\ ${ }^{1}$ Facultad de Instrumentación y Ciencias Atmosféricas, Universidad Veracruzana, Xalapa, Veracruz, \\ México, ${ }^{2}$ Lab. de Ecofisiología Tropical, Depto. de Ecología Funcional, Instituto de Ecología, \\ Universidad Nacional Autónoma de México, Ciudad Universitaria, Deleg. Coyoacán, C.P. 04510, \\ México, D.F., México, E-mail: ²vlbarradas@ecologia.unam.mx
}

\begin{abstract}
Resumen
Con el fin de determinar si el agua recolectada artificialmente de la niebla en la zona central montañosa del Estado de Veracruz es apta para el consumo humano, se realizó un muestreo preliminar de enero a marzo del año 2010, periodo de mayor frecuencia de nieblas, para analizar sus aspectos físicos, químicos y biológicos y evaluar su calidad. Se encontró que el agua contiene metales pesados como el mercurio, organismos coliformes y cantidades altas de nitrógeno amoniacal, pero esto se puede solucionar aplicando un tratamiento de saneamiento para uso humano con un costo de 0.00341 USD L-1 que no incluye mano de obra ni infraestructura. Se discuten las causas que pueden originar su contaminación y se concluye que el agua recolectada de la niebla no es adecuada para el consumo humano, pero sí para la agricultura.
\end{abstract}

Palabras Clave: agua potable, coliformes, frentes fríos, metales pesados, nortes.

\section{Quality of fog-water artificially collected in the Pixquiac watershed, Veracruz, Mexico: preliminary results}

\begin{abstract}
In order to determine if artificially collected water fog in the mountainous located in central the region of Veracruz state is suitable for human consumption; a preliminary sampling was made, from January to March (2010), the period with more fog frequency. The objective was to analyze the physical, chemical and biological aspects concerning water quality. It was found that this water is not suitable for direct human consumption, since it contains heavy metals such as mercury, coliform organisms and high amounts of ammonia nitrogen, though it is suitable for direct use in agriculture. However, it is possible to apply a sanitation treatment for human use at a cost of 0.00341 USD L $^{-1}$ not including the labor cost neither the necessary infrastructure. Various causes can lead to this water pollution are discussed and it is concluded that collected fog water is not suitable for human consumption, but it might be used for agriculture.
\end{abstract}

Key Words: drinking water, coliforms, cold fronts, heavy metal, Northern winds. 


\section{INTRODUCCIÓN}

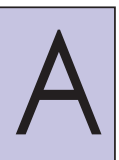
pesar de que el 75\% de la superficie terrestre está ocupada por agua, solamente el $2.76 \%$ es agua dulce y finalmente por su accesibilidad, sólo el $0.62 \%$ está disponible para el consumo humano que es agua subterránea y superficial ${ }^{1}$. En todas partes del mundo cada vez es más difícil obtenerla, por lo que en varios países se ha vuelto un grave problema social ${ }^{2}$.

En México, donde la distribución del agua dulce es radicalmente desigual, a su disponibilidad para consumo humano hay que agregar otros factores como el crecimiento poblacional y económico, que han ejercido mayor presión sobre las reservas de agua al momento que el volumen demandado es mayor que el suministrado en algunas regiones, lo que ocasiona problemas de distribución. La competencia por este recurso es actualmente causa de conflictos sociales de diferente intensidad y escala y se presenta tanto entre usuarios de la misma comunidad como en los de municipios, estados e incluso en el ámbito transfronterizo ${ }^{3}$.

En el Estado de Veracruz, a pesar de que el escurrimiento pluvial anual representa el 30\% del total nacional, no cuenta con sistemas de almacenamiento como presas y en la estación seca en algunas zonas el abasto es insuficiente ${ }^{4}$. Un ejemplo es la ciudad de Xalapa, que en la estación húmeda aparentemente no hay desabasto y en la temporada seca no sólo depende esencialmente del río Pixquiac que aporta el $38.25 \%$ de agua a la población, sino también del río Huitzilapan, Puebla ${ }^{5}$ aunque con algunos conflictos de suministro.

En la zona central montañosa del Estado de Veracruz incluida la microcuenca del río Pixquiac, existe una gran generación de niebla durante todo el año, siendo más notable en el periodo de noviembre a mayo, por lo que la captura artificial del agua a partir de la niebla puede ser un recurso hídrico alterno viable para la utilización y el consumo humano ${ }^{6,7}$.

Estudios anteriores han demostrado la factibilidad de la captura artificial de agua de la niebla en la región antes mencionada ${ }^{7-10}$. No obstante, a este tipo de agua no se le han hecho pruebas de su calidad. Por ello, se realizó un estudio preliminar, el primero en su género en la región, de la calidad y la cantidad de agua obtenida artificialmente de la niebla en la microcuenca, y si puede ser utilizada directamente en el consumo humano y/o en el riego agrícola o si es necesario la aplicación de un tratamiento para darle un uso adecuado.

\section{Materiales y mÉtOdos Sitio de estudio}

La captación de agua a partir de la niebla se realizó cerca del poblado de Mariano Escobedo, en un área despejada sin cobertura forestal $\left(19^{\circ} 29.914^{\prime} \mathrm{N}, 9^{\circ} 56.797^{\prime} \mathrm{O}, 1293 \mathrm{~m}\right.$ snm), en la microcuenca del río Pixquiac en la zona central montañosa del estado de Veracruz, México (Fig. 1), durante los meses de enero, febrero y marzo del 2010. El clima del área es el resultado de interacciones complejas entre los sistemas sinópticos dominantes, la topografía montañosa, la interacción planta-atmósfera y la proximidad al Golfo de México. En el verano el área se encuentra bajo los efectos de los vientos alisios a lo largo del extremo suroeste del anticiclón de las Bermudas $^{11}$, mientras que en el invierno, el anticiclón se sitúa cerca de Veracruz donde persiste una inversión por subsidencia que produce una capa de aire estratificada que se manifiesta como un banco de niebla entre 1,000 y 2,000 m snm ${ }^{12}$. La precipitación es altamente estacional con cerca del 80\% del total anual, presentándose en la estación húmeda (junio-julio a septiembre-octubre) y solamente el $20 \%$ en la estación seca (octubre-noviembre a mayo-junio). La temperatura y la precipitación media anual en el sitio de estudio es de $17.7^{\circ} \mathrm{C}$ y $1,706.3 \mathrm{~mm}$, respectivamente, y el promedio anual de días con niebla es de 44.2 (normales climatológicas correspondientes de los años 1981 a 2010), para los meses de diciembre y de enero a marzo (29.4), con 7.7, 8.3, 7.2 y 6.2 días en promedio, respectivamente, correspondientes a la estación climatológica de Briones $\left(19^{\circ} 30.5^{\prime} \mathrm{N}, 96^{\circ} 56.96^{\prime} \mathrm{O}, 1,349 \mathrm{~m} \mathrm{snm}\right)^{13}$.

\section{Recolección del agua de la niebla}

Para recolectar el agua de la niebla se utilizaron captadores en forma de rectángulo hechos de malla plástica (mosquitero de $3 \mathrm{~m}^{2}, 1 \mathrm{~m}$ de altura por $3 \mathrm{~m}$ de base con una separación del tejido de $1 \mathrm{~mm}$ ). A esta malla se le colocó en la base una canaleta de PVC de $5.08 \mathrm{~cm}$ de diámetro y de $3.2 \mathrm{~m}$ de largo con una manguera adherida a uno de los extremos, para que el agua captada de la niebla escurriera a través de la malla y se canalizara a un recipiente recolector, que cada vez que se desocupaba se desinfectaba y se lavaba con agua destilada. Se instalaron dos captadores como el descrito en el sitio de estudio (Figura 1), ubicando sus bases a una altura de $1.5 \mathrm{~m}$ de la superficie del suelo y perpendicularmente a la dirección dominante del viento (N-NE). Cerca de los captadores, pero en un área totalmente despejada, a una altura de $1.5 \mathrm{~m}$, se instaló un pluviómetro tipo balancín provisto de un microsistema de adquisición de datos (UA-003-64, HOBO, Bourne MA), para registrar la posible presencia de lluvia y sólo tomar en cuenta las muestras que no contenían este tipo de agua. También se instaló un captador de agua de la niebla tipo arpa, para medir independientemente la cantidad de agua capturada en cada muestreo, consistente de un cuadrado de $60 \times 60 \mathrm{~cm}$, con hilo sedal número 80 , con una separación de $2 \mathrm{~mm}$ entre hilos, presentando una área efectiva de captación de $0.36 \mathrm{~m}^{2}$. Este captador tipo arpa se instaló a $2 \mathrm{~m}$ de la superficie del suelo en una estructura techada, para aislarlo de la lluvia pero con libre paso de la niebla y conectado a un pluviómetro semejante al destinado a detectar la presencia de lluvia. Los pluviómetros se calibraron previamente generando un pulso por cada $0.2 \mathrm{~mm}$ de agua recolectada en el balancín y con ello los valores del 


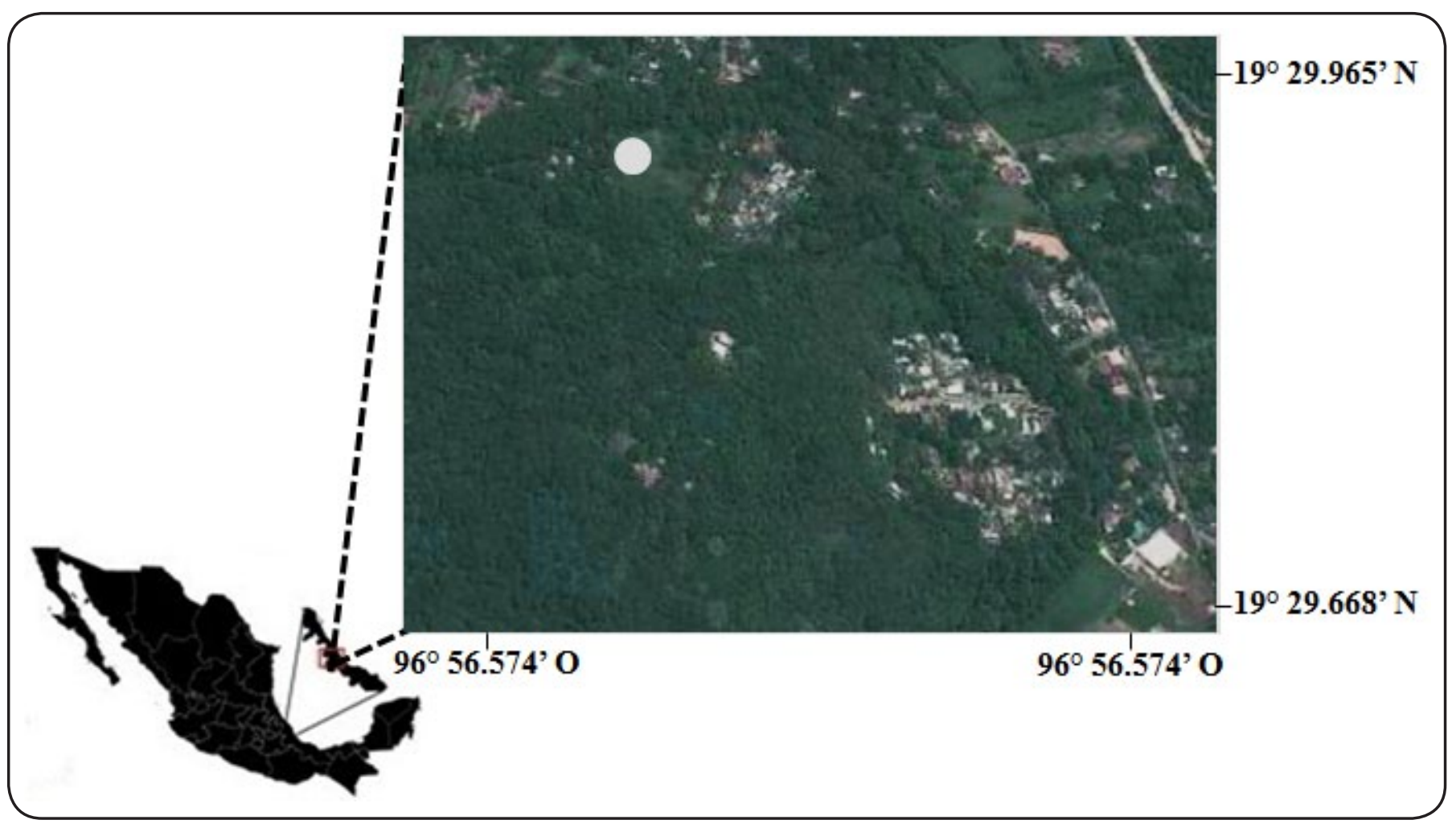

Figura 1. Localización del sitio donde se llevó a cabo la recolección de agua de la niebla (círculo blanco).

agua captada de la niebla se transformaron en el equivalente de precipitación pluvial (EPp).

\section{Análisis de las muestras de agua de la niebla}

Las muestras de agua de la niebla se analizaron en el Laboratorio de Alta Tecnología de Xalapa, S.C., de las que se determinó su color, turbiedad y composición química $(\mathrm{pH}$, nitratos, nitrógeno amoniacal, cloruros, dureza total $\left(\mathrm{CaCO}_{3}\right)$, sulfatos, As y $\mathrm{Na}$ ), metales pesados (Fe, $\mathrm{Mg}, \mathrm{Hg}, \mathrm{Pb}, \mathrm{Cd}, \mathrm{Cu}, \mathrm{Cr}$ y Zn) y composición biológica (organismos coliformes totales y fecales). En la Tabla I se muestran los elementos analizados, así como el método, su límite de detección y el equipo que se utilizó para determinarlos.

\section{Calidad del agua de la niebla}

La calidad del agua se determinó comparando los resultados de los análisis con la NOM-127-SSA1-1994 ${ }^{14}$, para su posible uso como agua potable y la NOM-001-ECOL-1996 ${ }^{15}$, para su posible uso como agua de riego agrícola. Esta última norma presenta promedios ponderados mensuales en función del volumen captado entre el tiempo de captación para las concentraciones de los elementos y el periodo de muestreo que fue de 36 días. Cabe señalar que la NOM-001-ECOL-1996 ${ }^{15}$ utiliza como parámetro el nitrógeno total, por lo que el resultado se reportó como la suma del nitrógeno amoniacal y el nitrógeno de nitratos, para saber si el agua podía usarse en el riego de cultivos, los valores encontrados en el agua se compararon con los límites máximos de metales pesados, coliformes fecales y huevos de helminto en agua de riego estipulados en la norma, ya que en ésta se establecen los lineamientos para la aplicación y certificación de buenas prácticas agrícolas y de manejo en los procesos de producción de frutas y hortalizas para consumo en fresco, que toma como fuente la parte de promedio mensual del uso en riego agrícola de la columna de ríos en la Tabla II de la NOM-001ECOL-1996 ${ }^{15}$, y en consideración a lo expuesto se realizaron los cálculos utilizando la siguiente ecuación ${ }^{16}$ :

$$
\bar{X}_{w}=\frac{\sum_{i=1}^{n} \mathrm{w}_{i} \mathrm{x}_{i}}{\sum_{i=1}^{n} \mathrm{w}_{i}}
$$

donde $\bar{X}_{w}$ es el promedio ponderado en función del volumen captado entre el tiempo de captación de los valores de cada muestra, $x_{i}$ son los resultados de cada muestra y $w_{i}$ es el volumen captado entre el tiempo de captación de cada muestra.

\section{Resultados \\ Estado atmosférico general en el periodo de recolección de agua de la niebla}

La Tabla II presenta la descripción de los fenómenos meteorológicos (frentes fríos, masa de aire frío, vaguadas, 


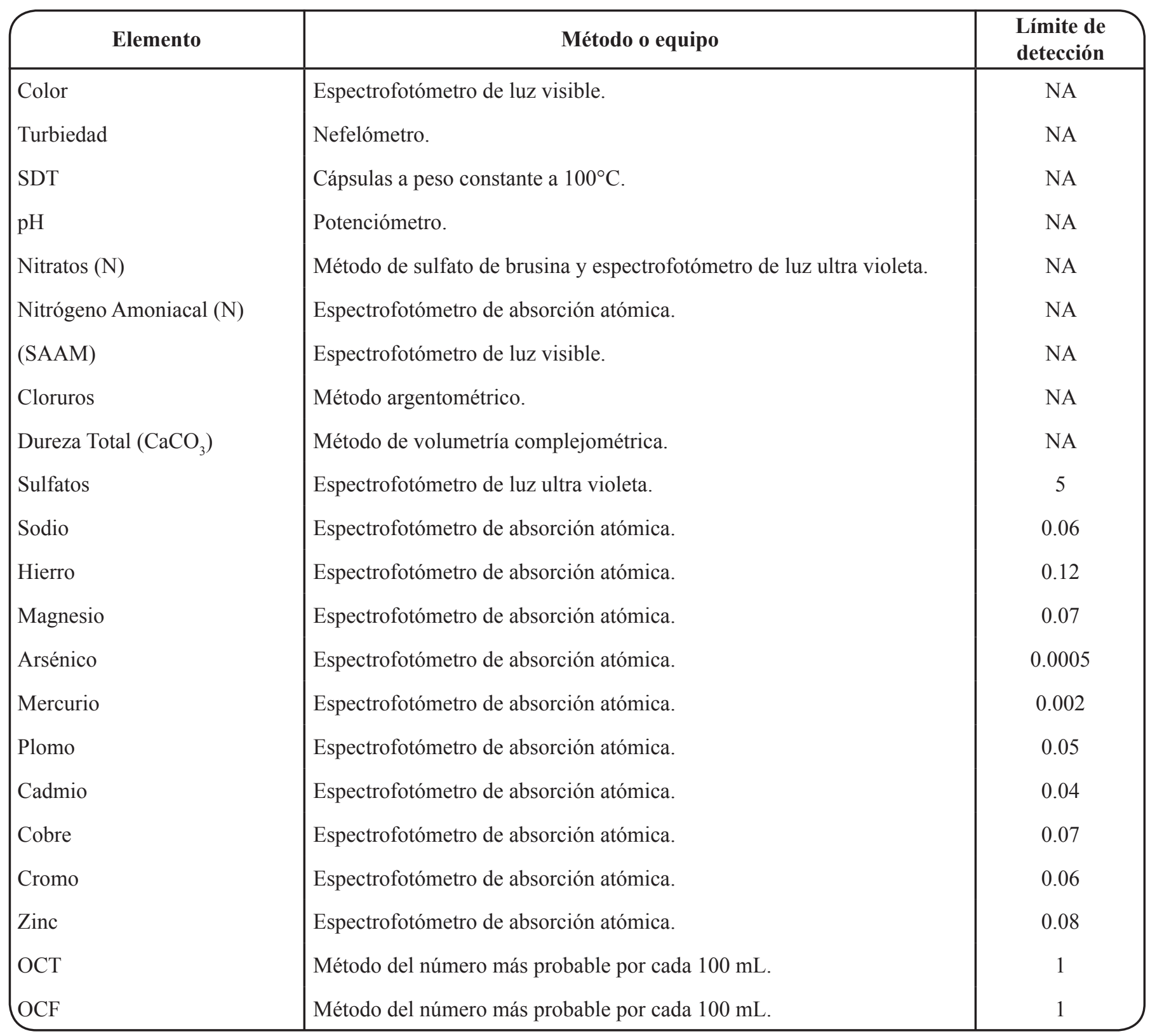

Tabla I. Elementos muestreados y equipo o método utilizado para su determinación (Sólidos disueltos totales, SDT; Sustancias Activas al Azul de Metileno, SAAM; Organismos Coliformes Totales, OCT; Organismos Coliformes Fecales, OCF).

posición de la corriente de vientos máximos, sistemas de alta presión), que se presentaron en la zona de estudio durante el período de muestreo y la estimación del volumen recolectado de agua de la niebla, por el captador tipo arpa. Es evidente que el 25 y 26 de enero del año 2010 , periodo que corresponde a la muestra 2 , se recolectó la mayor cantidad de niebla (3.19 mm día $\left.{ }^{-1}\right)$, siendo en promedio la cantidad total de agua obtenida durante las mediciones de $1.16 \mathrm{~mm}$ día $\mathrm{a}^{-1}$ expresado en equivalente de precipitación pluvial, no obstante, esta cantidad expresada como agua interceptada por el captador tipo malla fue de $66.0 \mathrm{~L} \mathrm{~m}^{-2}$ dentro del periodo de mediciones.

\section{Características físicas, químicas y biológicas del agua de la niebla para uso humano}

En la Tabla III se muestran los resultados obtenidos de los análisis por muestra y los límites máximos permitidos de cada elemento en el agua potable según la NOM-127-SSA1-1994 ${ }^{14}$, con lo que se determinó si las concentraciones exceden o no los límites permitidos. 


\begin{tabular}{|c|c|c|c|}
\hline Muestra & Fecha & Fenómeno meteorológico & EPp \\
\hline 1 & $\begin{array}{l}16 / 01 / 2010 \\
17 / 01 / 2010\end{array}$ & $\begin{array}{l}\text { Frente frío con corriente de vientos máximos } \\
\text { Sistema de alta presión y masa de aire frío } \\
\text { Corriente de vientos máximos }\end{array}$ & $3.1 \mathrm{~mm}$ \\
\hline 2 & $\begin{array}{l}25 / 01 / 2010 \\
26 / 01 / 2010\end{array}$ & $\begin{array}{l}\text { Sistema de alta presión y masa de aire frío } \\
\text { Sistema de alta presión y masa de aire frío }\end{array}$ & $6.38 \mathrm{~mm}$ \\
\hline 3 & $\begin{array}{l}27 / 01 / 2010 \\
29 / 01 / 2010 \\
30 / 01 / 2010 \\
31 / 01 / 2010 \\
01 / 022010 \\
02 / 02 / 2010\end{array}$ & $\begin{array}{l}\text { Sistema de alta presión y masa de aire frío } \\
\text { Frente frío y corriente de vientos máximos } \\
\text { Frente frío y corriente de vientos máximos } \\
\text { Sistema de alta presión y masa de aire frío } \\
\text { Corriente de vientos máximos } \\
\text { Sistema de alta presión y masa de aire frío } \\
\text { Corriente de vientos máximos } \\
\text { Vaguada y corriente de vientos máximos }\end{array}$ & $1.6 \mathrm{~mm}$ \\
\hline 4 & $\begin{array}{l}03 / 02 / 2010 \\
04 / 02 / 2010 \\
05 / 02 / 2010\end{array}$ & $\begin{array}{l}\text { Vaguada y corriente de vientos máximos } \\
\text { Frente frío y corriente de vientos máximos } \\
\text { Sistema de alta presión y masa de aire frío } \\
\text { Corriente de vientos máximos }\end{array}$ & $8.04 \mathrm{~mm}$ \\
\hline 5 & $\begin{array}{l}10 / 02 / 2010 \\
12 / 02 / 2010 \\
13 / 02 / 2010 \\
16 / 02 / 2010 \\
17 / 02 / 2010 \\
18 / 02 / 2010\end{array}$ & $\begin{array}{l}\text { Sistema de alta presión y masa de aire frío } \\
\text { Frente frío y corriente de vientos máximos } \\
\text { Sistema de alta presión y masa de aire frío } \\
\text { Sistema de alta presión y masa de aire polar } \\
\text { Sistema de alta presión y masa de aire frío } \\
\text { Corriente de vientos máximos } \\
\text { Sistema de alta presión y masa de aire frío } \\
\text { Corriente de vientos máximos }\end{array}$ & $6.43 \mathrm{~mm}$ \\
\hline
\end{tabular}

Tabla II. Fenómenos meteorológicos que se presentaron durante el tiempo de recolección de agua de la niebla y equivalente en precipitación pluvial (EPp) del recolector de niebla tipo arpa.

Durante la recolección se obtuvieron seis tipos diferentes de coloración del agua excediendo el límite máximo de la norma, como el caso de la muestra cinco con valores tan altos de hasta 55 UC y también estuvieron por arriba de los valores permitidos los resultados de las pruebas físicas, químicas y biológicas, el $\mathrm{pH}$ fue más ácido con un valor muy bajo de 6.2, sin embargo, hay que tomar en cuenta que para la lluvia el nivel mínimo de $\mathrm{pH}$ es de 5. Asimismo, los valores de concentración de amoniaco de las muestras de la 1 a la 3 fueron altos. Las muestras 4 y 5 resultaron positivas para mercurio e igual se exceden a lo establecido. Todas las muestras resultaron positivas para SAAM (Sustancias Activas al Azul de Metileno), pero como no se cuantifica en los resultados, por tanto, no se puede determinar si excede o no a la Norma Oficial Mexicana. Por otro lado, los análisis biológicos dieron por resultado la presencia de coliformes totales en las muestras 1,3 y 5 y presentaron valores por encima de la norma y para coliformes fecales sólo la muestra 1 resultó muy por encima de lo permitido, mientras que en otros muestreos no hubo presencia de estas bacterias. El resultado es que no es posible utilizar el agua proveniente de la niebla en esa zona del centro del Estado de Veracruz, sin embargo, existe la posibilidad de purificarla con un costo aproximado de $\$ 3.41 \mathrm{USD} \mathrm{m}^{-3}$ (15 de julio del 2010). 


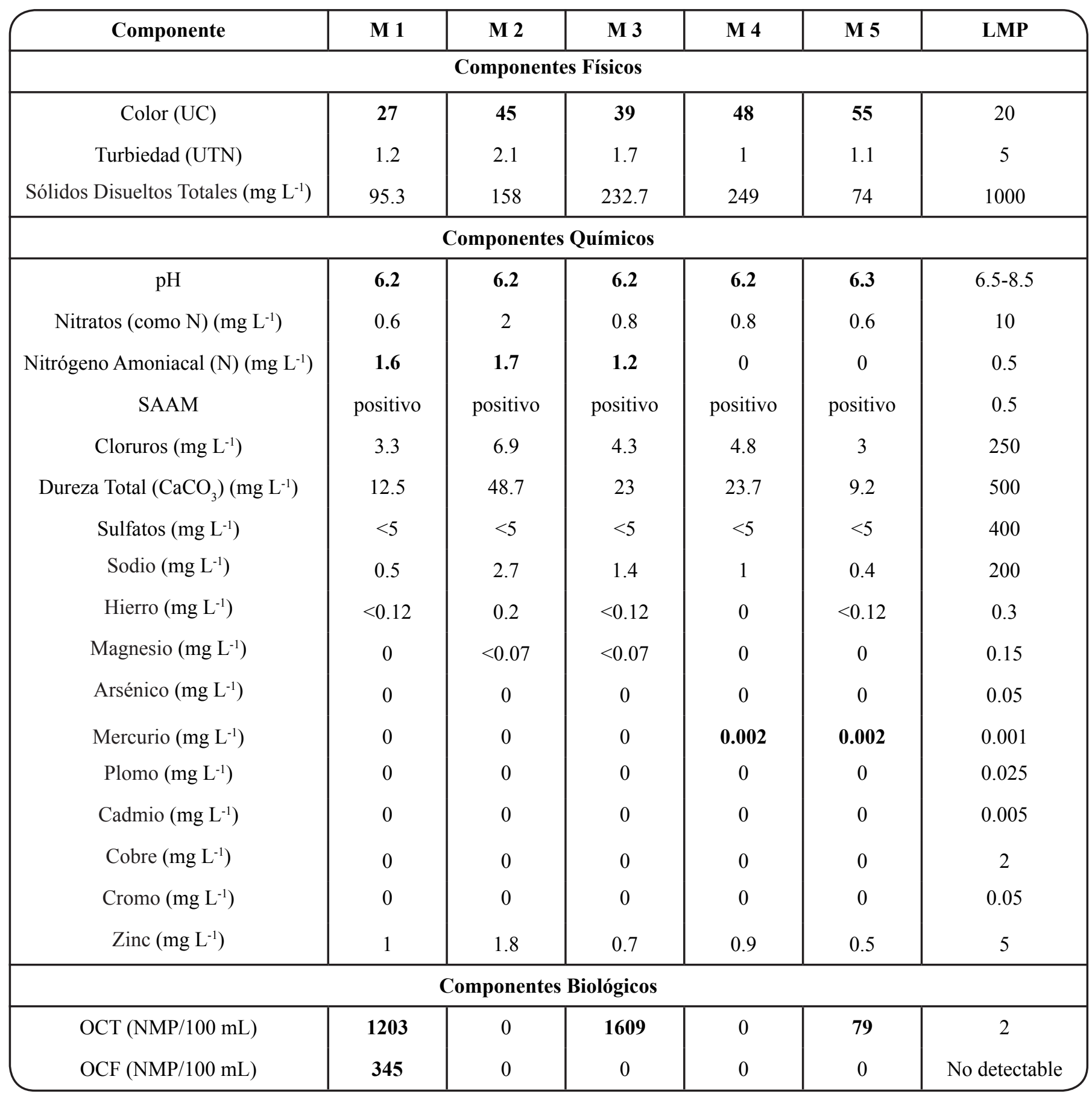

Tabla III. Concentración en cada muestra (M) de los componentes analizados y límites máximos permitidos (LMP) para el agua potable. Sólidos disueltos totales; Sustancias Activas al Azul de Metileno, SAAM; Organismos Coliformes Totales, OCT; Organismos Coliformes Fecales, OCF (en negritas todos los valores que exceden la Norma).

\section{Características físicas, químicas y biológicas del} agua para uso agrícola

La Tabla IV muestra los promedios ponderados mensuales de las cinco muestras analizadas, así como los límites máximos permitidos de cada contaminante para el agua de riego agrícola que determina la NOM-001-ECOL-1996 ${ }^{15}$ y si fueron detectados, saber si exceden o no el límite establecido. Ninguno de los componentes físicos, químicos y biológicos excedieron los valores que establece la norma para el agua de riego. 


\begin{tabular}{|c|c|c|c|}
\hline $\begin{array}{l}\text { Elemento } \\
\text { (unidades) }\end{array}$ & $\begin{array}{c}\text { Promedio ponderado } \\
\text { mensual }\end{array}$ & $\begin{array}{c}\text { Límite máximo permitido para } \\
\text { agua de riego (promedio mensual) }\end{array}$ & Estatus \\
\hline \multicolumn{4}{|c|}{ Pruebas Químicas } \\
\hline $\mathrm{pH}$ & 6.22 & $5-10$ & No excede \\
\hline Nitrógeno total $(\mathrm{mg} / \mathrm{L})$ & 2.05 & 40 & No excede \\
\hline Arsénico (mg/L) & 0 & 0.2 & No se detectó \\
\hline Mercurio (mg/L) & 0.000995 & 0.01 & No excede \\
\hline Plomo (mg/L) & 0 & 0.5 & No se detectó \\
\hline Cadmio (mg/L) & 0 & 0.2 & No se detectó \\
\hline Cobre (mg/L) & 0 & 4 & No se detectó \\
\hline Cromo $(\mathrm{mg} / \mathrm{L})$ & 0 & 1 & No se detectó \\
\hline Zinc (mg/L) & 1.1 & 10 & No excede \\
\hline \multicolumn{4}{|c|}{ Pruebas Biológicas } \\
\hline $\begin{array}{c}\text { Organismos Coliformes Fecales } \\
(\mathrm{NMP} / 100 \mathrm{~mL})\end{array}$ & 50 & 1000 & No excede \\
\hline
\end{tabular}

Tabla IV. Concentración promedio de los elementos analizados, límites máximos permitidos para el agua de riego y estatus de los elementos con respecto a estos límites. los coliformes fecales se reportan en número más probable/100 mililitros (NMP/100 mL), el pH en escala de potencial de hidrógeno y los demás elementos en miligramos/litro (mg/L).

\section{Análisis de precursores de contaminación atmosférica durante el periodo de muestreo}

La mayor cantidad de precursores de contaminación que generaron precipitaciónácida provinieron de las muestras 2,4 y 5 , y los precursores de contaminación por polvo, humo o aerosoles se presentaron en las muestras 2, 3, 4 y 5 y los precursores de contaminación por materia biológica correspondieron a las muestras 1,3 y 5 . El pH de las muestras de agua de la niebla fueron de la 1 a la 4 con 6.2 y la 5 con 6.3 que no es ácido para el agua de lluvia.

\section{Discusión}

La cantidad de agua recolectada de la niebla artificialmente en el sitio de estudio, primera vez que se realiza en la región, fue más baja que las encontradas en otros estudios de recolección natural de agua de la niebla, donde este mecanismo de interceptación fue producido por árboles de pino, tanto por un individuo como por el bosque ${ }^{6,10}$. Esto se debe a que los captadores artificiales no están diseñados para alcanzar la máxima captura de agua y su tamaño de $3 \mathrm{~m}^{2}$ no es comparable a los de los árboles; sin embargo, por las características físicas de las gotas que forman la niebla en la región ${ }^{8}$ se pueden diseñar captadores más eficientes para mejorar las cosechas de agua, aunque la cantidad total recolectada artificialmente en el tiempo de muestreo es bastante alta ya que representa el $40 \%$ de la precipitación pluvial en esa época (enero-febrero).

Algunos de los componentes encontrados en los análisis de las muestras de agua captada de la niebla excedieron la Norma Oficial Mexicana para el agua de consumo humano, pero no es común que aparezcan en el agua de la niebla ${ }^{17,18}$. Es posible que el comportamiento de los fenómenos meteorológicos dominantes en la región transporten diferentes contaminantes, principalmente el viento (nortes y suradas), emitidos por la termoeléctrica de Poza Rica, Veracruz $\left(20^{\circ} 31.98^{\prime} \mathrm{N}, 97^{\circ}\right.$ 27.0' O), ubicada al norte del sitio de estudio, así como los producidos por una cementera en Orizaba, Veracruz $\left(18^{\circ}\right.$ $\left.51^{\prime} \mathrm{N}, 97^{\circ} 06^{\prime} \mathrm{O}\right)^{19,20}$, al sur del sitio de estudio y llegaran a la zona de experimentación por el medio antes mencionado, producido por la llegada de un frente frío ${ }^{21}$ (frentes fríos 29 y 31 de la temporada invernal años 2009 y 2010). Lo anterior sí es posible como ha quedado demostrado con el mercurio, que una vez presente en la atmósfera, puede viajar cientos de miles de kilómetros contaminando lugares lejanos de los sitios donde la contaminación fue generada originalmente ${ }^{22}$.

Las concentraciones de nitrato de origen antropogénico fueron similares a las que presenta la lluvia en El Tajín $\left(20^{\circ} 26.88^{\prime} \mathrm{N}\right.$, 
$97^{\circ} 22.7^{\prime}$ O) y La Mancha $\left(19^{\circ} 44.13^{\prime} \mathrm{N}, 96^{\circ} 32.38^{\prime} \mathrm{O}\right)^{23}$, que como en el caso del mercurio pudieron haber sido generados por contaminación atmosférica producida en Poza Rica, Veracruz, además de la posible interacción de los vehículos de combustión interna de los poblados cercanos ${ }^{24} \mathrm{o}$ de la misma ciudad de Xalapa, Veracruz $\left(19^{\circ} 32.4^{\prime} \mathrm{N}, 96^{\circ} 55.65^{\prime} \mathrm{O}\right)$. El nitrato encontrado en las muestras 4 y 5 coinciden con las muestras donde se detectó el mercurio probablemente procedente de Poza Rica y es factible que la presencia de nitrógeno amoniacal, bacterias coliformes fecales y totales, así como substancias SAAM, sea por la contaminación de los captadores de la niebla tipo malla producida por pesticidas y/o fertilizantes, además de la posible descomposición de insectos o plantas y aminoácidos provenientes de ellos y excrementos de aves u otros animales.

Los resultados de los análisis indican que no es posible utilizar el agua proveniente de la niebla directamente para el consumo humano en esa zona del centro del Estado de Veracruz, pero existe la posibilidad de purificarla a un costo grosso modo de \$3.41 USD m ${ }^{-3}(15$ de julio del 2010), sin contar la mano de obra e infraestructura necesaria, con lo que el agua tendría un costo de 0.00341 USD L $^{-1}$, que no es competitivo con el que abastece al sector popular la Comisión Municipal de Agua Potable de Xalapa, Veracruz y tiene un valor de $\$ 0.00048$ USD L $^{-1}{ }^{[25]}$.

Si el agua recolectada de la niebla se utilizara para riego agrícola como en un sembradío de maíz, en un cálculo burdo de cosecha de agua, se requerirían sólo $2 \mathrm{~m}^{2}$ del captador tipo malla que capturaría $2.06 \mathrm{~L} \mathrm{~m}^{-2} \mathrm{día}^{-1}\left(66.0 \mathrm{~L} \mathrm{~m}^{-2}\right.$ del 16 de enero al 18 de febrero), si el maíz requiere $4 \mathrm{~mm}^{-1} \mathrm{a}^{-1}$ de precipitación (600 $\mathrm{mm}$ de precipitación aproximadamente en 5 meses), sin tomar en cuenta otros factores que pueden afectar el riego.

Esta agua también puede ser depositada/inyectada en el suelo donde llevaría su curso natural de purificación y que podría alimentar los mantos freáticos y restablecer toda aquella que se ha perdido por la deforestación de la región ${ }^{6,7}$, pero este mecanismo deberá ser validado con pruebas de campo. Además es necesario realizar más mediciones de cantidad y calidad de agua recolectada de la niebla en aquellos meses en que la frecuencia de nieblas es alta (de noviembre a abril, con valores de 4.3 a 8.3 día mes $^{-1}$ y un promedio de 6.33 día mes $^{-1}$ ) e incluir otros sitios en el gradiente altitudinal de la región.

Aunque la recolección de agua de la niebla se haya realizado en un solo sitio del extenso sistema montañoso del Estado de Veracruz, es posible establecer la hipótesis de que las concentraciones de contaminantes en el agua de la niebla serán muy semejantes en gran parte de la región, que se encuentra en la zona de influencia de los sistemas atmosféricos descritos. Sin embargo, es deseable continuar con este tipo de estudios, ya que la cosecha del agua de la niebla puede ser una fuente importante que puede compensar y mitigar la demanda de agua urbana.

\section{Conclusiones}

El agua de la niebla que se captó durante el periodo de muestreo puede ser utilizada como agua de riego sin necesidad de recibir algún tratamiento, pero para que pueda utilizarse en el consumo humano debe someterse a tratamientos de purificación con un costo muy alto comparado con la que suministra el municipio a la población.

El periodo donde se presentaron más tipos de precursores de contaminación fue durante la recolección de la muestra 5 (10 días). El tipo de precursor de contaminación que más se presentó durante el muestreo fue probablemente el debido al polvo, humo o aerosoles.

El desplazamiento de las masas de aire muy frío provenientes del norte, que al encontrarse con las masas de aire de la región (cálidas y muy húmedas), ocasionaron las nieblas más intensas/ densas generando una mayor recolección de agua.

Es evidente la necesidad de seguir realizando estudios de la calidad y cantidad del agua captada de la niebla, no sólo en el sitio de estudio, sino en otras zonas de la región montañosa del Estado de Veracruz o de las regiones de México donde la frecuencia de nieblas permitan una recolecta significativa de agua.

\section{Agradecimientos}

Los autores agradecen el financiamiento de esta investigación al Fondo Mixto CONACyT-Gobierno del Estado de Veracruz mediante el proyecto "Gestión integral del agua en la microcuenca del río Pixquiac" con número 37137.

\section{Referencias}

1. Fetter, C.Applied Hydrogeology (Macmillan Publishing Company, New York, 1991).

2. Hinrichsen, D., Robey, B. \& Upadhyay U.D. Solution for a watershort world (The Johns Hopkins University School of Public Health, Baltimore, 1998).

3. Saade, L. in El desafío para las políticas públicas de las reformas de segunda generación (Instituto Tecnológico Autónomo de México, México, D.F., 2001).

4. CONAGUA in http://siga.conagua.gob.mx/presas/geopresas.html (Consultada: febrero, 2015).

5. Contralínea. in http://www.veracruz.contralinea.com.mx/ archivo/2005/julio/htm/ guerra.htm (Consultada en febrero, 2015, 2005).

6. Barradas, V.L. Capacidad de captación de agua a partir de la niebla en Pinus montezumae Lambert de la Región de las Grandes Montañas del Estado de Veracruz, México. Biótica 8, 427-431 (1983).

7. Barradas, V.L. La importancia de la niebla como fuente natural y artificial de agua en la Región de las Grandes Montañas del Estado de Veracruz. Foresta Veracruzana 2, 43-48 (2000).

8. García-García, F. \& Montañez, R.A. Warm fog in eastern Mexico: A case study. Atmósfera 4, 53-64. 
9. Anaya Bautista, J.A.Evaluación preliminar de la captura artificial de niebla en la microcuenca del río Pixquiac (Tesis de Licenciado en Ciencias Atmosféricas, Facultad de Instrumentación Electrónica y Ciencias Atmosféricas, Universidad Veracruzana. Xalapa, Veracruz, 2010) 42 págs.

10. Sánchez Falfán, A. Captación el agua a partir de la niebla por pinos en la región montañosa central de Veracruz (Tesis de Licenciado en Ciencias Atmosféricas, Facultad de Instrumentación Electrónica y Ciencias Atmosféricas, Universidad Veracruzana. Xalapa, 2011) 100 págs.

11. Alemán, P.A.M. \& García, E. in World survey of climatology (ed. Landsberg, H.) 345-391 (Elsevier, Amsterdam, 1974).

12. Barradas, V.L., Cervantes-Pérez, J., Ramos-Palacios, R., PuchetAnyul, C., Vázquez-Rodríguez, P. \& Granados-Ramírez, R. in Tropical Montane Cloud Forests (eds. Bruijnzeel, L.A., Scatena, F.N. \& Hamilton, L.S.) 549-556 (Cambridge University Press, Cambridge, 2010).

13. SMN in http://smn.cna.gob.mx/index.php?option=com content $\&$ view $=$ article $\& i d=42 \&$ Itemid $=75$ (Consultada en diciembre, 2014).

14. Secretaría de Salud. Norma Oficial Mexicana NOM-127 SSA11994, salud ambiental, agua para uso y consumo humano. Límites permisibles de calidad y tratamientos a que debe someterse el agua para su potabilización (Secretaría de Salud, Ciudad de México, 1994) Pág. 15.

15. Secretaría de Medio Ambiente y Recursos Naturales. Norma Oficial Méxicana NOM-001-SEMARNAT-1996. Límites máximos permisibles de contaminantes en las descargas de aguas residuales en aguas y bienes nacionales (Secretaría de Medio Ambiente y Recursos Naturales, Ciudad de México, 1996) Pág. 5.

16. Madansky, A. \& Alexander H.G.B. Weighted standard error and its impact on significance testing (The Analytical Group, Inc., Scottsdale, AZ, 2008).
17. Cereceda, P., Schemenauer, R.S. \& Suit, M. Producción de agua de niebla en Perú. Alisios, 3, 63-74 (1993).

18. Cereceda, P., Larrain, H., Lázaro, P., Osses, P., Schemenauer R.S. \& Boroevic X. La niebla, agua potable para zonas rurales. Revista Geográfica de Chile Terra Australis, 45, 143-160 (2000).

19. Báez, A., Padilla, H., Cervantes, J. Pereyra, D. \& Belmont, R. Rainwater chemistry at the eastern flanks of the Sierra Madre Oriental, Veracruz, México. Journal of Geophysical Research 102, 23329-23336 (1997).

20. Walcek, C., De Santis, S. \& Gentile T. Preparación de un inventario de emisiones de mercurio para el este de América del Norte. Environmental Pollution 123, 375-381 (2003).

21. Ledesma, M. \& Baleriola, G. Meteorología aplicada a la aviación (Thompson-Paraninfo, España, 2002).

22. Naciones Unidas. Estudio global sobre mercurio incrimina a los incineradores de residuos (Programa de las Naciones Unidas para el Medio Ambiente, Washington, 2003).

23. Marín, A. H., Bravo, A. Alarcón, A. L. Sosa R. \& Sánchez P. Presencia de lluvia ácida en el Estado de Veracruz y el uso de modelos de trayectoria inversa para identificar fuentes de emisión de precursores. Casos de estudio: El Tajín y La Mancha. in Memorias XIX Congreso Nacional de Meteorología y IV Congreso Internacional de Meteorología, OMMAC. 17 al 23 de Octubre, Saltillo Coahuila, México (2010).

24. Hoyer, M., Baldauf, R., Scardbro, C., Barres, J. \& Keeler, G. Mercury Emissions from Motor Vehicles. 13th International Emission Inventory Conference Working for Clean Air in Cleanwater. Clearwater, FL, June 8-10. Available at: www. epa.gov./glnpo/nsdocs/hgsbook/ (2003).

25. CMAS-Xalapa (Comisión Municipal de Agua Potable y Saneamiento, Xalapa, 2015, http://www.cmasxalapa.gob.mx/ pdf/Tarifas_Febrero_2015.pdf). 